\title{
Video In The Shadow Of An Object To Determine When And Where Of The Video
}

\author{
An Qianwen
}

\author{
School of electrical and electronic engineering, North China Electric Power University, Baoding \\ 071000, China. \\ 765456234@qq.com
}

Keywords:Least square optimization,Nonlinear equations,Genetic Algorithm

\begin{abstract}
Shadows of the sun position techniques can determine the place and date of the video. First, use Matlab to extract video files, extract $\mathrm{n}$ pictures at such intervals, after reading the picture get $\mathrm{n}$ sets of the shadow length $\mathrm{l}$ changes with time $\mathrm{t}$. Known date from capture date calculate solar declination, using least-squares optimization model, each parameter the mathematical relationship was established, the longitude and latitude as an unknown parameter nonlinear fitting, fitting location for Inner Mongolia Erdos (110.52 ${ }^{\circ}$ East longitude, latitude $39.89^{\circ}$ ); Unknown date, the question will be transformed into longitude and latitude, declination angle of nonlinear equations three unknowns, using genetic algorithms to find possible locations for Inner Mongolia Erdos (longitude 109.36, $40.16^{\circ}$ North latitude), capture date is June 22.
\end{abstract}

\section{Introduction}

How to determine video shot on location and shoot date is an important aspect of analyzing video data, the sun shadow location technology is through the analysis of video objects in the shadows of the Sun changes, one way to determine the place and date of video capture. This paper will discuss the known date and date taken unknown cases, how to determine location.

Gives a straight pole change of shadows in the Sun video, straight bar and have some way to estimate the height of 2 meters, according to video filming locations and dates are determined.

According to the height of bars in video, shadow and the actual bar height is determined by the ratio of the long shadow of different time bar. In the case of known date taken, videos shot on location can be determined the declination of the Sun, solar altitude, true solar time and the calculation formula can be get straight shadow function for a long time; Will include a video shot on location longitude and latitude these two unknown parameters of the function equation for Nonlinear least square optimization problems, using the Matlab function lsqnonlin in the location's longitude, latitude, the optimal solution. In the case of unknown date, under known conditions with longitude, latitude, declination of nonlinear equations three unknowns, using genetic algorithms, and possible locations.

\section{The least-squares optimization method to determine the parameters of models}

\subsection{Model assumptions}

1) video bar perpendicular to the horizontal ground.

2) bars are fine enough, ignore the straight RADIUS.

3) videos give time to GMT.

4) obtained using two-dimensional picture, straight shadow length straight bar real shadow long. 


\begin{tabular}{cc}
\hline Symbols & definitions \\
\hline$(\alpha, \beta)$ & Longitude and latitude of straight bars \\
$\mathrm{h}$ & Straight bar height \\
$\mathrm{l}$ & Bar length of shadow \\
$\mathrm{H}$ & Sun elevation angle \\
$\mathrm{A}$ & Sun azimuth \\
$\delta$ & Solar declination \\
$\theta$ & Hour angle \\
$\mathrm{t}$ & True solar time \\
$\mathrm{n}$ & Measurement date (n of days a year)
\end{tabular}

\subsection{Solving parameters based on least square optimization model}

1) preparation of the model

Sun Solar altitude angle $\mathrm{h}$ and solar azimuth angle a two angles. Sun azimuth A: is the Sun projected on the ground level and the angle between the local Meridian, that is, the direction of the Sun's horizontal projection the angle of deviation from the South. Sun elevation angle $\mathrm{H}$ : is the direction of sunlight, and the angle between the ground plane $\left(0^{\circ} \leq \mathrm{H} \leq 90^{\circ}\right)$. Solar declination $\delta$ : is the latitude of the Sun, the Earth's equatorial plane and the angle between the Sun and Earth centre line. The angle $\theta$ : angle is 0 when the local time is 12 o'clock when, before and after every hour, an increase of $360 \% / 24=15^{\circ}$. True solar time t: is the hour angle of true Sun looking circular Planar Centre measured.

Set bar height and shadow lengths of $\mathrm{h}$ and $\mathrm{l}$ respectively, bar the height and length of the shadow in the video were h' and l' respectively. According to proportion, The shadow length straight bar for

$$
l=\frac{h}{h^{\prime}} l^{\prime}
$$

Video is a straight bar in Beijing 8:54-9:34 changes in the shadow of the Sun. Use MATLAB to read video, collecting an image every 4 minutes; take advantage of reading image analysis collecting image, get the bar top, bottom and straight shadow the position coordinates of the vertex. Straight fixed and perpendicular to the ground, so the length of the rod in the video $h$ ' for the straight bar vertices with straight bottom $y$-coordinate difference to calculate $h^{\prime}=670$. Video shadow length is the distance between bars to a shadow culmination at the bottom, according to reading data from images, Video straight length of shadow l' is calculated, according to equation (1), the Beijing bar that corresponds to the shadow length $\mathrm{l}$, the results are shown in table 1.

Table 1: straight bar the sun shadow length

\begin{tabular}{ccc}
\hline Beijing time & Video straight length of & Actual bar length of shadowl $/ \mathrm{m}$ \\
\hline $08: 54$ & 810.08 & 2.42 \\
$08: 58$ & 788.03 & 2.35 \\
$09: 02$ & 769.02 & 2.30 \\
$09: 06$ & 747.00 & 2.23 \\
$09: 10$ & 731.00 & 2.18 \\
$09: 14$ & 709.01 & 2.12 \\
$09: 18$ & 691.04 & 2.06 \\
$09: 22$ & 672.05 & 2.01 \\
$09: 26$ & 655.10 & 1.96 \\
$09: 30$ & 636.10 & 1.90 \\
$09: 34$ & 621.08 & 1.85 \\
\hline
\end{tabular}


2) model and solution

According to the bar height of $h=2 m$, date of video $n=194$, Beijing time $t 0=8: 54-9: 34$, longitude and latitude of straight bars $(\alpha, \beta)$ these 5 parameters, write 5 equations about bars shadow length $l$, solar altitude angle $\mathrm{H}$, solar declination $\delta$, the true solar time $\mathrm{t}$ and hour angle $\theta$.

$$
\left\{\begin{array}{c}
h=l \tan H \\
\sin H=\sin \beta \sin \delta+\cos \beta \cos \delta \cos \theta \\
\delta=23.45 \sin \left(\frac{2 \pi(284+n)}{365}\right) \\
t=t_{0}-\left(120^{\circ}-\alpha\right) \times 4 \\
\theta=15^{\circ} \times(12-t)
\end{array}\right.
$$

Sorting system of nonlinear equations (2), contain two unknown parameters of video shot on location longitude and latitude, straight shadow length 1 function of Beijing time t0

$$
\frac{h}{\sqrt{h^{2}+l^{2}}}=\sin \beta \sin \delta+\cos \beta \cos \delta \cos \left[15 \times\left|12-\frac{t-\left(120^{\circ}-\alpha\right) \times 4}{60}\right|\right]
$$

Table 1 straight bar shadow length $l$ and its corresponding time t0 into equation (3), by using the least-squares optimization method in the lsqnonlin function, get (3) of unknown parameters $\alpha$ and $\beta$ in the optimal solution.

Obtain video shot on location longitude latitude and alpha beta are as follows: 110.52 and 39.89 degrees. The latitude/longitude coordinates, Ordos city, Inner Mongolia is (111.23, $39.87{ }^{\circ}$ ) calculated result was very close, Ordos city, latitude and longitude coordinates, so the video shot on location, Ordos city, Inner Mongolia.

\section{The genetic algorithm parameter model}

\section{1. preparation of the model}

Genetic algorithm is a kind of evolutionary algorithms, its basic principle is to follow the laws of biological evolution, the problem parameter encoded as a chromosome, using iterative methods of selection, crossover and mutation operations to chromosomes in the population information, ultimately generate the target chromosome.

1). Coding:

GA in searching solution spaces before the data represents the genotype data into genetic space, different combinations of these data will constitute a different point.

Initial groups of build: $n$ initial randomly generated string data structure. GA to the $n$ series of structured data as the initial point of evolution. Fitness assessment: the fitness of individuals or the pros and cons of solutions.

2). Select:

Selection aims to elect from the current group of good individuals, so that they have the opportunity to be a parent on behalf of the next generation of breeding children. Selection principle is adaptable to grab the probability of individual contributions for the next generation of offspring. Cross: get new individual by doing the cross, embodies the idea of exchange of information. Variations: the first randomly selected individuals in the group, for selected individuals with a certain probability of data is changing the structure of each string in value.

3).The termination conditions:

When the best individual fitness reaches a given threshold, or best individual fitness and population fitness is no longer rising, or the number of iterations reaches a preset algebra, algorithm terminates.

\section{2. model and solution}

For a given date, using the genetic algorithms to solve the possible locations and dates.Based on 
the nonlinear equation (2), and use longitude $\alpha$ 、 latitude $\beta$ and solar declination $\delta$ the three unknown parameters to build nonlinear equations

$$
\frac{h}{\sqrt{h^{2}+l_{i}^{2}}}=\sin \beta \sin \delta+\cos \beta \cos \delta \cos \left[15 \times\left|12-\frac{t_{i}-\left(120^{\circ}-\alpha\right) \times 4}{60}\right|\right], i=1,2, \cdots, 10
$$

Among them, the h-bar high, TI, Li long shadow. The nonlinear equations is overdet-ermined system of equations, using conventional methods to solve more difficult, so I chose to use genetic algorithms to solve them.

Order

$$
\left\{\begin{array}{c}
f_{1}\left(l, t_{1}\right)=\frac{h}{\sqrt{h^{2}+l_{1}^{2}}}-\sin \beta \sin \delta+\cos \beta \cos \delta \cos \left[15 \times\left|12-\frac{t_{1}-\left(120^{\circ}-\alpha\right) \times 4}{60}\right|\right] \\
f_{2}\left(l, t_{2}\right)=\frac{h}{\sqrt{h^{2}+l_{2}^{2}}}-\sin \beta \sin \delta+\cos \beta \cos \delta \cos \left[15 \times\left|12-\frac{t_{2}-\left(120^{\circ}-\alpha\right) \times 4}{60}\right|\right] \\
\vdots \\
f_{10}\left(l, t_{10}\right)=\frac{h}{\sqrt{h^{2}+l_{10}^{2}}}-\sin \beta \sin \delta+\cos \beta \cos \delta \cos \left[15 \times\left|12-\frac{t_{10}-\left(120^{\circ}-\alpha\right) \times 4}{60}\right|\right] \\
f(l, t)=f_{1}^{2}\left(l, t_{1}\right)+f_{2}^{2}\left(l, t_{2}\right)+\cdots+f_{10}{ }^{2}\left(l, t_{10}\right)
\end{array}\right.
$$

Solving systems of nonlinear equations into optimization problems $\min [\mathrm{f}(\mathrm{x})]$.

By calling GADST Matlab genetic algorithm Toolbox, set the size to 5000, maximum number of evolved for 1000 , calculated the optimal solution for: longitude and latitu-de $\left(109.36^{\circ}, 40.16^{\circ}\right)$ 、 declination angles23.43 ${ }^{\circ}$, longitude and latitude $\left(109.36^{\circ},-40.16^{\circ}\right)$ 、 declination angles-23.43 . Located place in the Inner Mongolia Ordos and India Ocean, capture date is June 22. According to video shown in landscape, video shooting location for the India Ocean is not consistent with the actual situation, so the video shot on location, Ordos city, Inner Mongolia.

\section{Summary}

In two cases of known and unknown date obtained video shot on location for Erdos, that seeks to place reasonable. Taken at unknown dates, get the shoot date is June 22, while the actual filming date is July 13, date taken so get large errors. Model in the case of known or unknown date taken, based on video objects in the shadows of the Sun changes more accurately determine the location of video and can probably guess video filmed at the time.

\section{Reference}

[1] Declination angles, http: // baike .baidu. com /link ?url =vteF Xim oBwToZW-5NV4Euq _xVLNcNxvF WK qbYD o4-K 0avh 8XpU-IW U5y-ND_JVl9xoG ogQvVHczD8Yi3JCxnAK, 2015-10-29.

[2] Sun azimuth, http://baike.haosou.com/doc/6120265-6333412.html, 2015-10-29.

[3] Sun elevation angle, http://baike.haosou.com/doc/4128005-4327467.html, 2015-10-29.

[4] Hour angle, http://baike.haosou.com/doc/6834673-7051893.html, 2015-10-29.

[5] True solar time, http://baike.haosou.com/doc/5334896-5570334.html, 2015-10-29.

[6]ShiFeng, ect. Intelligent algorithm for analysis of 30 cases, Beijing: Beijing University of 
Aeronautics and Astronautics press, 2010, 57-59.

[7] Si Shoukui, Sun Xi cyanine. mathematical modeling and application, Beijing: national defense industry press, 2014, 95.

[8] Wang Bingzhong, encyclopedia of lessons of solar radiation, 2011, 1-6.

[9] Xu Jianwei, astronomical algorithms, 2009, 1-305. 